PHYSICAL REVIEW D 93, 084042 (2016)

\title{
Can we measure individual black-hole spins from gravitational-wave observations?
}

\author{
Michael Pürrer, ${ }^{1,2}$ Mark Hannam, ${ }^{2}$ and Frank Ohme ${ }^{2}$ \\ ${ }^{1}$ Max Planck Institute for Gravitational Physics (Albert Einstein Institute), \\ Am Mühlenberg 1, D-14476 Potsdam-Golm, Germany \\ ${ }^{2}$ School of Physics and Astronomy, Cardiff University, \\ Queens Building, CF24 3AA Cardiff, United Kingdom \\ (Received 18 December 2015; published 20 April 2016)
}

\begin{abstract}
Measurements of black-hole spins from gravitational-wave observations of black-hole binaries with ground-based detectors are known to be hampered by partial degeneracies in the gravitational-wave phasing: between the two component spins, and between the spins and the binary's mass ratio, at least for signals that are dominated by the binary's inspiral. Through the merger and ringdown, however, a different set of degeneracies apply. This suggests the possibility that, if the inspiral, merger and ringdown are all within the sensitive frequency band of a detector, we may be able to break these degeneracies and more accurately measure both spins. In this work we investigate our ability to measure individual spins for nonprecessing binaries, for a range of configurations and signal strengths, and conclude that in general the spin of the larger black hole will be measurable (at best) with observations from Advanced LIGO and Virgo. This implies that in many applications waveform models parameterized by only one effective spin will be sufficient. Our work does not consider precessing binaries or subdominant harmonics, although we provide some arguments why we expect that these will not qualitatively change our conclusions.
\end{abstract}

DOI: $10.1103 /$ PhysRevD.93.084042

\section{INTRODUCTION}

The Advanced LIGO (aLIGO) [1] and Advanced Virgo $(\mathrm{AdV})[2]$ detectors carry the potential to observe hundreds of black-hole-binary systems per year by the time they reach design sensitivity (2019-2020) [3-5]. Each binary is characterized by the black-hole masses and spins, which we hope to measure from gravitational-wave (GW) observations. However, we expect partial degeneracies in the dependence of the waveform on these parameters to limit the accuracy of their measurement [6-8].

We can learn about the constituents of the binary system through the phasing of the binary as the two objects orbit and slowly spiral toward each other. The phasing is a function of the black-hole masses and the individual spin vectors. For a system where the spins are aligned with the binary's orbital angular momentum we may parametrize the binary by the masses $m_{1}$ and $m_{2}$ and the dimensionless spin parameters $\chi_{1}$ and $\chi_{2}$, where the Kerr limit imposes $\left|\chi_{i}\right|<1$. During the inspiral, the leading-order postNewtonian (PN) influence of the spins arises as a weighted sum of $\chi_{1}$ and $\chi_{2}[7,9]$ :

$$
\chi=\frac{m_{1} \chi_{1}+m_{2} \chi_{2}}{M}-\frac{76 \eta}{226}\left(\chi_{1}+\chi_{2}\right)
$$

where $M=m_{1}+m_{2}$ is the total mass and $\eta=m_{1} m_{2} / M^{2}$ is the symmetric mass ratio. Equation (1.1) implies a strong degeneracy between $\chi_{1}$ and $\chi_{2}$, so that even when $\chi$ can be measured accurately, it will be difficult to measure the individual spins. This degeneracy is strongest for equalmass systems. At large mass ratios, $\chi$ is dominated by the spin of the larger black hole, so in these cases we may be able to measure the large black hole's spin, but the spin of the smaller black hole will be poorly measured, if at all. There is also a partial degeneracy between $\chi$ and the mass ratio of the two black holes, which limits our ability to measure the individual masses and $\chi[6-8,10]$.

When the spins also have components lying in the orbital plane, the binary's orbital plane precesses and the GW signal acquires further structure $[11,12]$. There are again degeneracies in the effect on the phasing, and in this case the mean influence on the orbital precession of the four inplane spin components during the course of the inspiral can be combined into a single "effective precession spin" parameter [13]. Once again, these degeneracies suggest that we may be able to measure the spin of the binary's larger black hole, but it will be difficult to accurately measure both black-hole spin vectors.

The preceding discussion was restricted to the black holes' inspiral. During the merger and the final black hole's ringdown, the GW amplitude and phase are parameterized by the final black hole's mass and spin, which in turn are governed by a different combination of the progenitor masses and spins. It is conceivable that in observations of high-mass systems, where the inspiral and mergerringdown contribute comparable power to the detectable signal, that we may be able to more tightly constrain the individual masses and spins. A preliminary study of this question was performed in Ref. [14]. No evidence was 
found that two-spin effects would be measurable in advanced-detector observations, but the study was limited to a small number of configurations, and at the time there was no two-spin inspiral-merger-ringdown (IMR) model available with which to systematically estimate parameter measurement uncertainties. We now have in hand a twospin model that has been proposed for aligned-spin binaries, SEOBNRv2 [15], which makes it possible to address this question more thoroughly.

Here we use a simplified single-detector Bayesian framework to systematically analyze the spin information that can be inferred from observing binary mergers. Although we do not perform an exhaustive study, and cannot rule out the possibility that particularly favorable configurations do exist, our basic conclusion is that only one spin parameter can be constrained by GW observations, and single-spin models are sufficient for the needs of GW astronomy during the advanced-detector era.

Our study is limited to aligned-spin systems, because these are the only configurations for which a two-spin IMR model exists that are fast enough to make parameter estimation studies feasible. Nonetheless, we perform some preliminary studies of precessing systems (in the inspiral regime) and make some comments in Sec. IV on how well we expect our results to carry over to generic binaries.

We first present our methodology in Sec. II, which summarizes the Bayesian techniques we use to measure the binary parameters, the SEOBNRv2 waveform model, and our Markov-chain-Monte Carlo (MCMC) code. We then move on to our results in Sec. III and discuss our conclusions in Sec. IV.

\section{METHODOLOGY}

\section{A. Posterior probability}

In order to assess our ability to measure the source parameters $\theta$ from an observation, we perform the following study. We simulate a GW signal from a binary with parameters $\theta_{0}$ and confront it with a variety of signals exploring the full parameter space of possible sources. Assuming stationary Gaussian noise of the instrument, we are interested in the expected, noise-averaged parameter recovery. Hence we set the noise realization to zero [i.e., our data only consist of the GW signal $\left.h\left(\theta_{0}\right)\right]$ while we use the instrument's noise spectral density $S_{n}$ in the inner product between two signals:

$$
\left\langle h(\theta) \mid h\left(\theta_{0}\right)\right\rangle=4 \operatorname{Re} \int_{f_{\min }}^{\infty} \frac{\tilde{h}(f ; \theta) \tilde{h}^{*}\left(f ; \theta_{0}\right)}{S_{n}(f)} d f .
$$

Here, $\tilde{h}$ denotes the GW signal in the Fourier domain, ${ }^{*}$ is the complex conjugation, and we use the aLIGO "zero-detuned high power" design sensitivity [16] with $f_{\min }=10 \mathrm{~Hz}$ throughout this paper.
Given the data $d \equiv h\left(\theta_{0}\right)$, the likelihood ratio between the hypothesis that a signal with parameters $\theta$ is contained in $d$, or $d$ is pure Gaussian noise, is [17]

$$
\Lambda=\frac{\exp \left(-\left\langle h\left(\theta_{0}-h(\theta)\left|h\left(\theta_{0}\right)-h(\theta)\right\rangle / 2\right)\right.\right.}{\exp \left(-\left\langle h\left(\theta_{0}\right) \mid h\left(\theta_{0}\right)\right\rangle / 2\right)} .
$$

We simplify Eq. (2.2) by expanding the linear inner product and maximizing the likelihood over the template norm $\rho=\sqrt{\langle h(\theta) \mid h(\theta)\rangle}$. By straightforward differentiation of Eq. (2.2), we find $\rho_{\max }=\rho_{0}\left\langle\hat{h}\left(\theta_{0}\right) \mid \hat{h}(\theta)\right\rangle$ and

$$
\hat{\Lambda}=\max _{\rho} \Lambda=\exp \left(\frac{\rho_{0}^{2}}{2}\left\langle\hat{h}(\theta) \mid \hat{h}\left(\theta_{0}\right)\right\rangle^{2}\right) .
$$

Here, $\rho_{0}=\sqrt{\left\langle h\left(\theta_{0}\right) \mid h\left(\theta_{0}\right)\right\rangle}$ is the simulated signal-tonoise ratio (SNR) and

$$
\hat{h}=\frac{h}{\sqrt{\langle h \mid h\rangle}}
$$

denotes the normalized waveform.

Bayes' theorem allows us to express the posterior probability of the source parameters, given the data, as the product of the likelihood $\hat{\Lambda}(\theta)$ with the prior probability $\pi(\theta)$ :

$$
\mathcal{P}(\theta)=\frac{\hat{\Lambda}(\theta) \pi(\theta)}{\mathcal{P}_{0}},
$$

where $\mathcal{P}_{0}$ can be interpreted as a simple normalization factor such that

$$
\int \mathcal{P}(\theta) d \theta=1
$$

When we are interested in the posterior probability for individual parameters, we present the marginalized posterior by integrating over all parameters that we are not interested in. Formally, our results are then based on a mixture of a likelihood that was maximized over extrinsic parameters (distance, orientation, sky location) which all contribute to the template norm [cf. Eq. (2.3)] and a posterior that is marginalized over intrinsic parameters.

The intrinsic parameters we vary are the chirp mass $M_{c}$, the symmetric mass ratio $\eta$, the dimensionless, aligned-spin components $\chi_{i}(i=1,2)$, as well as a reference (or "coalescence") time $t_{c}$ and phase $\phi_{c}$ :

$$
\begin{aligned}
\theta & =\left\{M_{c}, \eta, \chi_{1}, \chi_{2}, t_{c}, \phi_{c}\right\}, \\
M_{c} & =\frac{\left(m_{1} m_{2}\right)^{3 / 5}}{(m 1+m 2)^{1 / 5}}, \\
\eta & =\frac{m_{1} m_{2}}{\left(m_{1}+m_{2}\right)^{2}}, \\
\chi_{i} & =\frac{\vec{S}_{i} \cdot \hat{L}}{m_{i}^{2}} .
\end{aligned}
$$


Here, $m_{i}$ are the individual black-hole masses, $\vec{S}_{i}$ are their spin vectors and $\hat{L}$ is the direction (unit length) of the orbital angular momentum. We only consider spins that are either aligned or antialigned with $\hat{L}$.

In order to optimize the calculation of $\mathcal{P}(\theta)$, we use the fact that we can marginalize analytically over $\phi_{c}$. The dominant mode of nonprecessing signals obeys

$$
h\left(\phi_{c}+\Delta \phi\right)=h\left(\phi_{c}\right) e^{-i 2 \Delta \phi},
$$

which allows us to express

$$
\begin{gathered}
\frac{1}{2 \pi} \int_{0}^{2 \pi} \hat{\Lambda} d \phi_{c}=\frac{1}{2 \pi} \int_{0}^{2 \pi} e^{8 \rho_{0}^{2} \cos ^{2}\left(2 \phi_{c}\right)|\mathcal{O}|^{2}} d \phi_{c} \\
=e^{4 \rho_{0}^{2}|\mathcal{O}|^{2}} I_{0}\left(4 \rho_{0}^{2}|\mathcal{O}|^{2}\right),
\end{gathered}
$$

where $I_{0}$ is the Bessel function of the first kind and $\mathcal{O}$ is the complex integral that is part of the inner product:

$$
\mathcal{O}=\int_{f_{\min }}^{\infty} \frac{\tilde{h}(f) \tilde{h}_{0}^{*}(f)}{S_{n}(f)} d f .
$$

In addition, we can efficiently marginalize over the time $t_{c}$ by noting that

$$
\tilde{h}\left(t_{c}+\Delta t\right)=\tilde{h}\left(t_{c}\right) e^{i 2 \pi f \Delta t} .
$$

Therefore, we can calculate $\mathcal{O}$ for a range of time shifts by using fast algorithms for the discrete inverse Fourier transformation. By summing the results over time, we effectively marginalize over $t_{c}$ as well, which means our codes only have to sample the physical mass and spinrelated parameters. A similar strategy has been described by Farr in an internal technical document [18].

We note that maximizing over time and phase is computationally even faster, and our code is able to perform either the maximization or marginalization over time and phase efficiently as a result of the above calculations. Since we found virtually indistinguishable results with both methods, we used time- and phase-maximized posteriors in the majority of cases we report here.

\section{B. MCMC code}

We use the ensemble sampler EMCEE [19] together with the LALSuite [20] library that contains the implementation of the waveform models discussed in Sec. II C.

The simulations use the single-detector likelihood described in Eq. (2.3). This is based on the inner product (2.1) which we calculate as a discrete inverse Fourier transformation (see the discussion in the previous section). We discretize the integral with a frequency spacing of $\Delta f=0.5 \mathrm{~Hz}$, which is larger than the inverse duration of some of the signals we consider. However, we only need to resolve the correlations between $h(\theta)$ and $h\left(\theta_{0}\right)$, and in numerical tests these turned out to have significant support only over a small range of time shifts if the optimally aligned waveforms agree well, which is the region we are interested in here. The same technique was recently used to speed up the construction of stochastic template banks [21]. In order to increase the time-domain resolution for maximization or marginalization over $t_{c}$, we use zero padding of the signal and template by a factor of 2 .

In general we use a setup of $\sim 10000$ iterations with 100 MCMC chains. In addition we have checked the robustness of our results with longer runs using ten MCMC chains evolved for $\sim 100000$ iterations. The first half of the samples is discarded to remove the burn-in of the chains which are not draws from the posterior distribution. We comine samples from chains that have a Gelman-Rubin statistic [22] close to unity. The above setup yields several thousand independent samples.

We choose uniform priors in the component masses with a minimum of $1.5 M_{\odot}$ for $m_{1}$ and $6 M_{\odot}$ for $m_{2}$. The upper cutoff is chosen to be several times the component mass of the signal mass parameters to leave ample room for sampling the mass space. We sample only half of the mass space, assume $m_{2} \geq m_{1}$ and add a cutoff in total mass $M_{\text {tot }} \leq 500 M_{\odot}$. The priors on the aligned spins are taken to be uniform in $\chi_{i} \in[-1,0.99]$.

In addition, two simulations with the precessing PN SpinTaylorT4 model as implemented in LALSuite [20] were performed with the nested sampling code from LALINFERENCE [23] for a three-detector aLIGO-AdV setup. We used a sampling rate of $4096 \mathrm{~Hz}$, no amplitude corrections, zero noise, the aLIGO [16] and AdV [24] design power spectral densities (PSDs) with a lower frequency cutoff of $40 \mathrm{~Hz}$ and a network SNR of 30 .

\section{Reduced-order model for SEOBNRv2}

To explore the measurability of aligned component spins for complete IMR waveforms over a large parameter space region we use a reduced-order model (ROM) [25] "SEOBNRv2_ROM" of the spin-aligned effective-onebody model "SEOBNRv2" [15]. At the moment SEOBNRv2 is the only two-spin IMR model available, but due to its high computational cost it cannot be used directly for parameter estimation studies that routinely require millions of likelihood evaluations. This study has only become feasible with the availability of the ROM [25] which is constructed using extensions to the techniques described in Ref. [26]. This ROM provides speedups on the order of several thousands over SEOBNRv2.

The SEOBNRv2 model was found to be accurate to a mismatch of $1 \%$ against $38 \mathrm{NR}$ waveforms from the SXS Collaboration [27], for total masses $M \in[20,200] M_{\odot}$ and the aLIGO design PSD [16]. Recent studies have shown that while SEOBNRv2 is extremely accurate, it disagrees 
with new BAM [28] and SXS [29] NR waveforms at high aligned spin $[30,31]$.

The ROM [25] has a worst mismatch against SEOBNRv2 of $\sim 1 \%$, but in general mismatches are better than $\sim 0.1 \%$. It covers the entire SEOBNRv2 parameter space $0.01 \leq \eta \leq 0.25$ and $-1 \leq \chi_{i} \leq 0.99$ for compact binaries of total mass $M_{\text {tot }} \geq 2 M_{\odot}$ and the full aLIGO design sensitivity starting at $10 \mathrm{~Hz}$.

We note that the LAL implementation [20] of SEOBNRv2 and SEOBNRv2_ROM only provide the $l=m=2$ mode of the GW signal. Currently, no models are available that include both spin and higher modes. Since we also use SEOBNRv2_ROM for the target signals we cannot investigate the effects of higher modes in this study.

The waveform used here also does not include effects of precession of the orbital plane and of the black-hole spins. Precession is driven by mainly a single measurable parameter $[13,32]$ that describes spin in the orbital plane. At total masses where the merger ringdown contributes significantly to the overall power, the number of precession cycles is very small. Therefore we do not expect precession to change the qualitative picture at those total masses, as we will discuss in Sec. IV.

\section{RESULTS}

We now use the methodology described in Sec. II to study the accuracy with which we can measure the individual spins of aligned-spin binaries. We focus on total masses where both the inspiral and merger-ringdown contribute a significant amount of power to the GW signal in aLIGO. The nominal mass we choose is $50 M_{\odot}$. If we make a crude split between inspiral and merger-ringdown at the Schwarzschild innermost stable circular orbit (ISCO) frequency, then at this mass a nonspinning equal-mass binary produces $60 \%$ of its detectable power during the inspiral and $40 \%$ during the merger and ringdown. We use the aLIGO "zero-detuned high power" design sensitivity [16] with a lower frequency cutoff of $f_{\min }=10 \mathrm{~Hz}$.

We consider mass ratios of $q=1,4$ and 10 and a range of (equal) aligned spins $\chi_{i}=-0.9,0,0.5,0.9$. We explore parameter recovery with two models: the double alignedspin SEOBNRv2 model and a single-spin SEOBNRv2 model, i.e., a model that assumes equal-spin waveforms $\chi_{1}=\chi_{2}$, which can be expressed in terms of the reduced spin parameter $\chi$. Instead of $\chi$ we present results in terms of a rescaled reduced spin [30]:

$$
\hat{\chi}:=\frac{\chi}{1-76 \eta / 113},
$$

which takes values in $[-1,1]$. We choose a SNR of 30 , which is considered optimistic but not unreasonable for aLIGO observations. Note that if we assume a uniform volume distribution of sources, and a threshold SNR of $\sim 10$ for detection, then since the SNR is proportional to the source distance, only $(10 / 30)^{3}$ of signals will be close enough to have a SNR higher than 30; i.e., $~ 96 \%$ of signals will have SNRs below 30. Similarly, only one in a hundred observations will have an SNR greater than 50 and one in a thousand greater than 100 .

Once we have presented results for our nominal choices (equal spins, $50 M_{\odot}$, and a SNR of 30), we consider the effect on our results of varying each of these in turn; we look at unequal spins in Sec. III B, the effect of varying the total mass in Sec. III C, and the dependence on SNR in Sec. III D, to determine at which SNR we may be able to constrain the spin on the smaller black hole.

\section{A. Equal-spin binaries}

Our key results for $50 M_{\odot}$ binaries are shown in Fig. 1. The left column of panels shows the posteriors for the reduced spin with respect to the symmetric mass ratio, while the right panel shows the posteriors for $\chi_{2}$ versus $\chi_{1}$. The plots show contours for $95 \%$ credible regions $\mathcal{C}$ of the marginal probability distribution function (PDF) which are a subset of the parameter space that includes $95 \%$ of the posterior probability, $\int_{\mathcal{C}} \mathcal{P}(\theta) d \theta=0.95$.

Much of what we can conclude about our ability to measure the individual black-hole spins can be inferred from this figure; the remainder of this paper will be devoted to demonstrating that the trends we observe here are generic. Our observations are as follows.

Consider first the panels on the left, which show the twodimensional posterior distribution for the reduced spin and mass ratio. The credible regions form strips. This is consistent with the degeneracy between mass ratio and spin that has been discussed in detail in previous work $[7,8,10,33]$. During the inspiral the approximate degeneracy is between the mass ratio and the reduced spin (see the discussion in Ref. [8]), while during the ringdown the degeneracy is between systems with the same final mass and spin. For the systems shown in Fig. 1, both degeneracies play a role. We will see in Sec. III C how these uncertainty regions vary with respect to total mass.

The tilt of the $\eta-\hat{\chi}$ posteriors depends on the spin and on the mass ratio. The regions are almost parallel to the $\eta$ axis for high aligned spins and have the largest slope for high antialigned spins. The variation in the tilt of the posteriors with respect to spin becomes more pronounced as we move to higher mass ratios. Thus, we can measure the reduced spin best for configurations with high aligned spin or equalmass systems and worst if the spin is highly antialigned and the system at a higher mass ratio. We note that this effect is due to the parameter dependence of the signals during the inspiral; we will see in Sec. III C that, as we move to highermass systems and more of the signal is from the merger and ringdown, these regions rotate.

Now consider the right-hand panels, which show the same results, but with $\chi_{1}$ plotted against $\chi_{2}$. The dashed lines indicate the results from using a single-spin model 

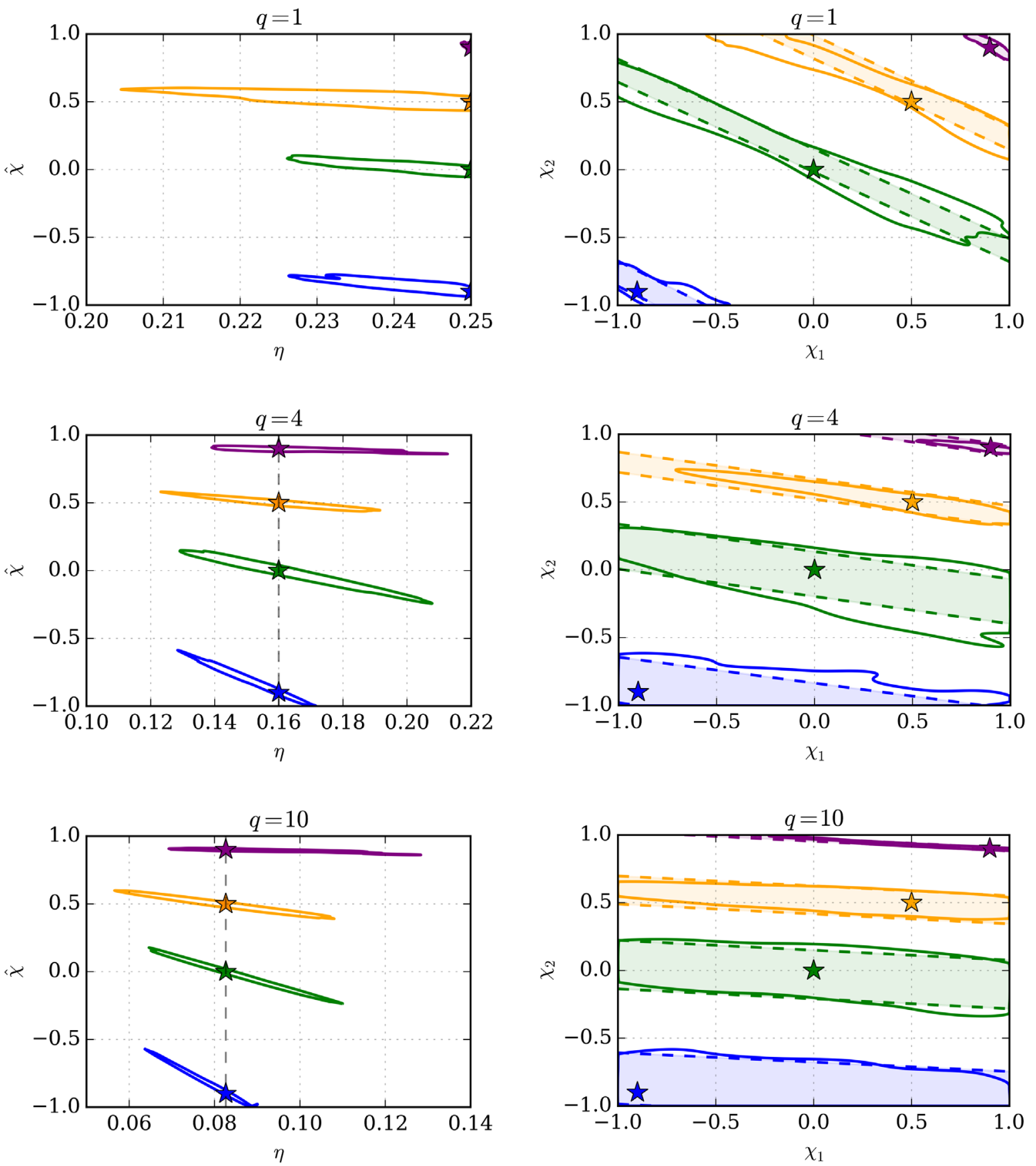

FIG. 1. Symmetric mass ratio $\eta$ versus rescaled reduced spin $\hat{\chi}$ (left), and component spin $\chi_{1}$ versus $\chi_{2}$ (right) posteriors for configurations with mass ratios $q=1,4,10$ (top to bottom) at total mass $50 M_{\odot}$ and SNR 30. Each panel shows configurations with equal aligned spins $\chi_{1}=\chi_{2}=-0.9,0,0.5,0.9$ (blue, green, orange, and magenta, respectively) with the true parameters indicated by a star symbol. The contours represent $95 \%$ credible regions. In the right column we show in addition to the $\chi_{1}-\chi_{2}$ samples colored bands that are delineated by two lines of constant reduced spin. These are obtained from the posteriors of the single-spin model at $95 \%$ credible level.

parameterized with respect to the effective spin; these lines represent constant values of the reduced spin, and their orientation arises purely from the definition Eq. (1.1). We now see that the individual spins are very poorly constrained, and the credible regions extend over the full spin range possible for each value of the reduced spin.
For equal-mass systems, lines of constant reduced spin are diagonal, and we measure each spin equally poorly. The spin measurements are constrained only by the Kerr limit. This means that we can only measure them accurately if they are both near extremal and with the same orientation; this is clear from the configurations with spins 
$\chi_{1}=\chi_{2}= \pm 0.9$. At the other extreme, if the reduced spin is small, as in the case where $\chi_{1}=\chi_{2}=0$, then the measurement is consistent with any magnitude of $\chi_{1}$ or $\chi_{2}$, with only the requirement that the other spin be of similar magnitude and in the opposite direction.

As the mass ratio increases, the spin of the larger black hole increasingly dominates the GW phasing, and this causes the lines of constant reduced spin to rotate. At high mass ratios, the spin of the larger black hole becomes a better approximation of the value of the reduced spin. At mass ratio $1: 10$, we see that we are now able to better measure the spin of the larger black hole; its uncertainty has been reduced to roughly a factor of 2 of the uncertainty in the reduced spin. The spin of the smaller black hole remains unconstrained, and in fact it is poorly constrained even for systems with a large reduced spin.

This tells us that our best hope of accurately measuring the spin of both black holes in a binary is for an equal-mass system with both spins near extremal and both aligned (or both oppositely aligned) to the orbital angular momentum. For high mass-ratio systems, or those with a small-tomoderate value of the reduced spin, the small black hole's spin is difficult to measure at all. However, for large massratio systems, we can measure the large black hole's spin, regardless of its value.

Based on the results from the single-spin model that are overlaid on the right-hand panels of Fig. 1, we may reasonably ask: what does a two-spin model tell us that we do not already learn from a simpler single-spin model, together with the constraints that follow from the definition of the reduced spin? The mass-ratio 1:4 results with moderate spins suggest that we will obtain a slightly stronger bound on the spin of the smaller black hole with a two-spin model, but the improvement is not dramatic.
We will return to this point in subsequent sections, where we consider systems with unequal spins, varying masses, and higher values of the SNR.

\section{B. Unequal spins}

So far we have restricted ourselves to configurations with equal aligned spins $\chi_{1}=\chi_{2}$. We have found that the reduced $\operatorname{spin} \chi$ is measured well, but the individual spins are not, beyond constraints implied by the Kerr limit. In this section we illustrate that the situation does not appear to change even if the spins are unequal.

As our example we consider three configurations at mass ratio $1: 4$, all with the same reduced spin $\chi=0$. These systems have a total mass of $50 M_{\odot}$. In one configuration both black holes have no spin, and in the others $\chi_{1}= \pm 0.75$, with $\chi_{2}$ chosen such that the reduced spin is still zero.

Figure 2 shows the two-dimensional 95\% credible regions for the component spins and histograms of the one-dimensional probability distributions of the spins. It is evident that the credible regions are almost identical. We first look at results for mass ratio $q=4$ (right panel). While the PDFs of the spins peak close to the true values, they all have almost the same support. In the presence of detector noise these differences are unlikely to be measurable. At mass ratio $q=1$ (left panel), the credible regions cover a similar region, but the peaks in the one-dimensional PDFs depend strongly on the black-hole spins of the signal. These results suggest that, while a single-spin model would provide almost no information about the individual spins in this case, a doublespin model would provide stronger evidence that the binary consists of either nonspinning black holes or highly spinning black holes with opposite orientations.
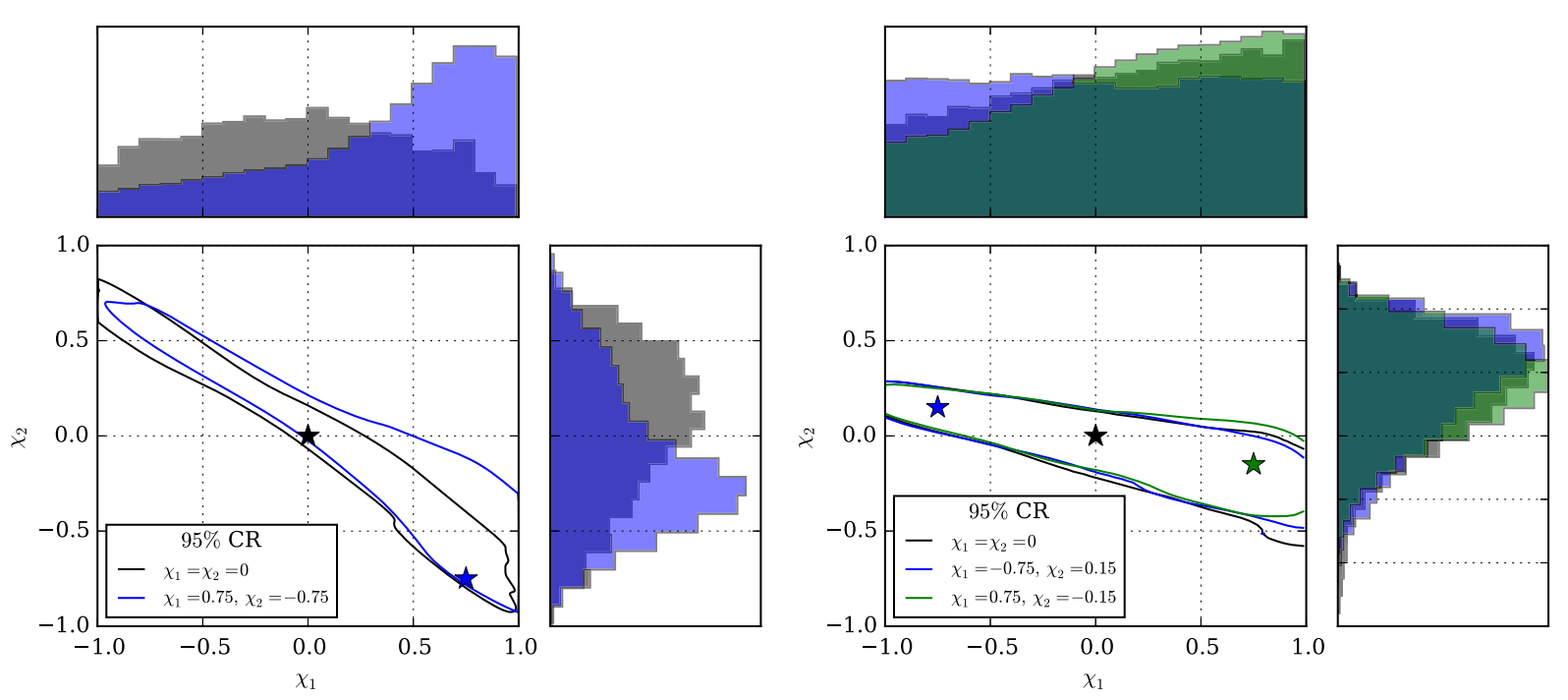

FIG. 2. The $95 \%$ credible regions for unequal spins, but identical reduced spin $\chi$. The configurations have SNR 30, and $M_{\text {tot }}=50 M_{\odot}$ and mass ratio $q=1$ (left) and $q=4$ (right). While the signal is symmetric under exchange of the masses for $q=1$, the prior is not. 

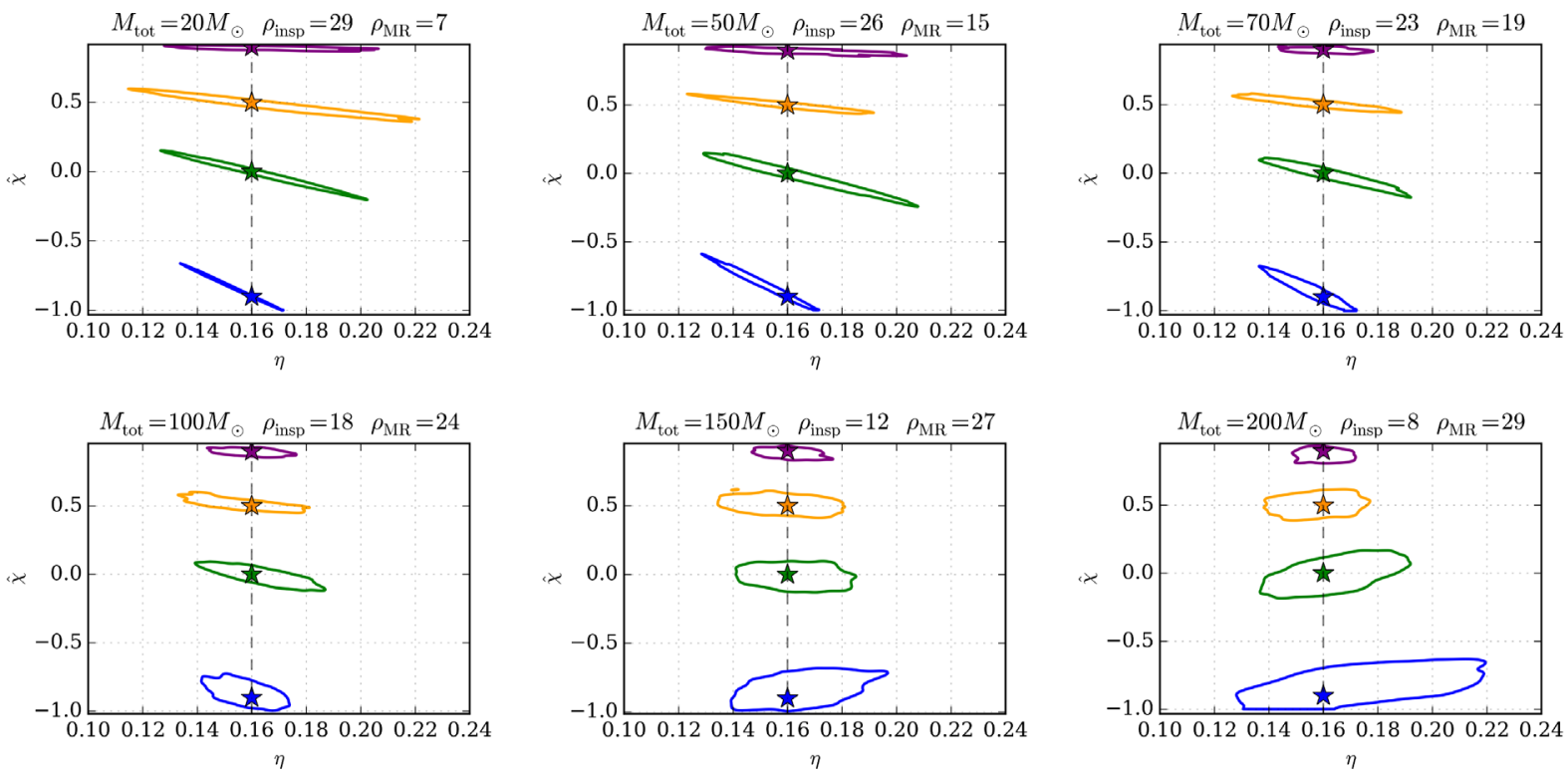

FIG. 3. The $95 \%$ credible regions for $\eta-\hat{\chi}$ posteriors for mass ratio $q=4$ and a range of total masses. We give SNRs in the inspiral (up to the Schwarzschild ISCO) and merger-ringdown (from the ISCO onwards) for nonspinning configurations. The total SNR is 30 in all cases.

\section{Dependence on total mass}

One of the motivations for this study was to explore to what extent the approximate reduced-spin degeneracy is affected when the GW detectors are sensitive to comparable power from both the inspiral and the merger-ringdown. This relative distribution of power is a function of the total mass of the system. In this section we investigate how the picture given in Sec. III A depends on the total mass and comment on the measurement accuracy of the mass ratio, reduced spin and component spins. The accuracy of recovery for chirp mass and total mass was studied in Refs. [34-36].

In Fig. 3 we show how the mass-ratio-reduced spin posteriors change with total mass. The correlation is very strong at low mass, with the posterior samples approximately following a straight line. As the mass increases the regions become more fuzzy and thicker, indicating a weaker correlation. This implies that measurement accuracy for $\chi$ decreases slightly if the posterior is almost parallel with the $\eta$ axis, i.e., for high aligned spins. If the spin is smaller, or the spins antialigned, the slope is large
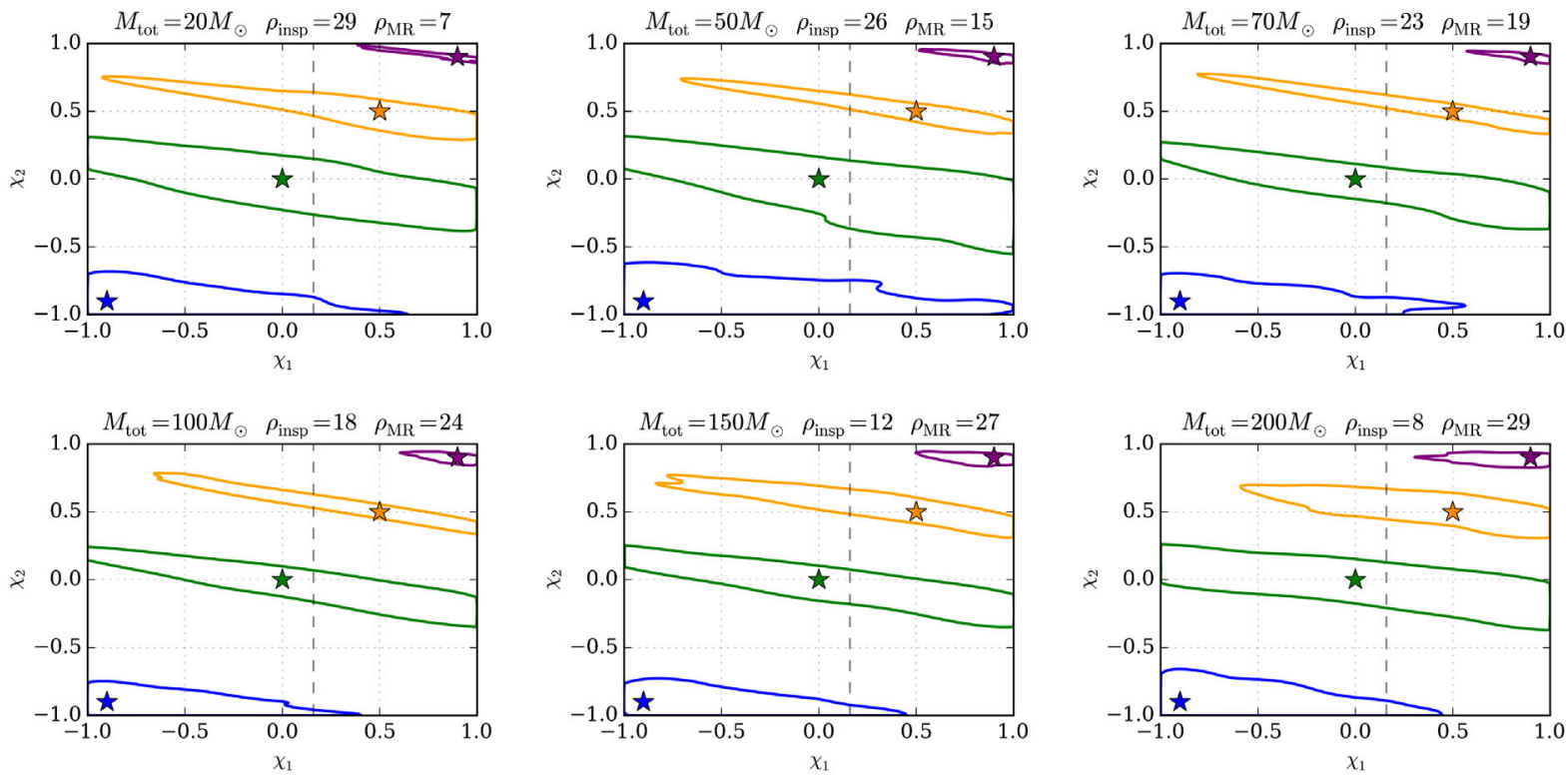

FIG. 4. The $95 \%$ credible regions for $\chi_{1}-\chi_{2}$ posteriors for mass ratio $q=4$ and a range of total masses. We quote SNRs as in Fig. 3 . 

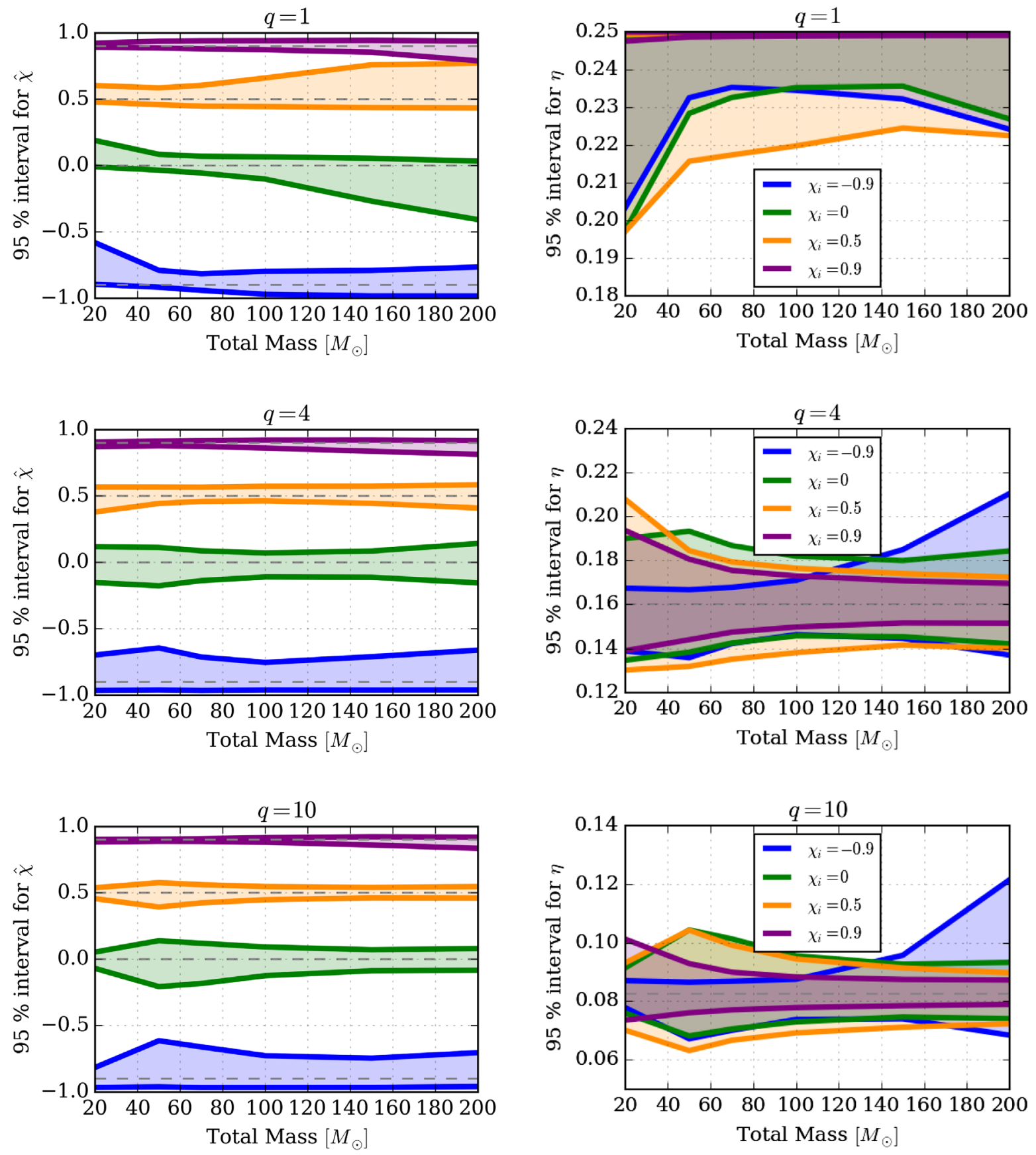

FIG. 5. The $95 \%$ intervals for the rescaled reduced spin $\hat{\chi}$ (left) and the symmetric mass ratio $\eta$ (right) as a function of total mass for a series of configurations at mass ratios $q=1,4,10$ (top to bottom).

enough that the increased width of the region does not greatly affect the $\chi$ measurement.

Figure 4 shows how the spin posteriors change with total mass. It is apparent that the posteriors do not significantly qualitatively change. Recent studies have pointed out that SEOBNRv2 is not expected to be accurate for high alignedspin systems at unequal mass [30,31]. Therefore our results for $\chi_{i}=0.9$ might change once an improved model is available.
To condense the information further the left panel of Fig. 5 shows the $95 \%$ credible interval for $\chi$ for mass ratios $q=1,4,10$. There is some broadening of the intervals for equal-mass systems at high total mass, but overall the reduced spin is pretty consistently well measured for a wide range of the parameter space. It is exceptionally well measured for high aligned spins.

The measurement accuracy of the symmetric mass ratio $\eta$ depends both on the total mass and on the spin. For high 
aligned spins the accuracy actually improves as the total mass is increased. For high antialigned spins the accuracy first improves with the total mass and is best around $M_{\text {tot }} \sim 70-100 M \odot$. It then worsens considerably if the mass is further increased. This becomes very obvious in the right panel of Fig. 5 for unequal mass systems. The behavior is reversed at equal mass.

\section{Dependence on SNR}

So far we have discussed results at a fixed SNR of 30. If the SNR is not too low, we can expect that the parameter measurement uncertainties scale inversely with the SNR [37]. The mass-ratio-spin degeneracy is not absolute, and at sufficient SNR we expect to be able to measure the spin of the smaller black hole. How high must the SNR be? We consider a nonspinning system to avoid the $\chi_{2}$ extremeKerr boundary. We choose a mass ratio $q=4$. At SNR 30 we cannot constrain the spin on the small black hole for this system, irrespective of the total mass.

We know from PN results that SNRs of hundreds to thousands are required to constrain the spin on the small black hole $[6,7,10,38]$. By including merger-ringdown we expect that we can constrain this spin at a lower SNR if the total mass is chosen so that inspiral and merger-ringdown

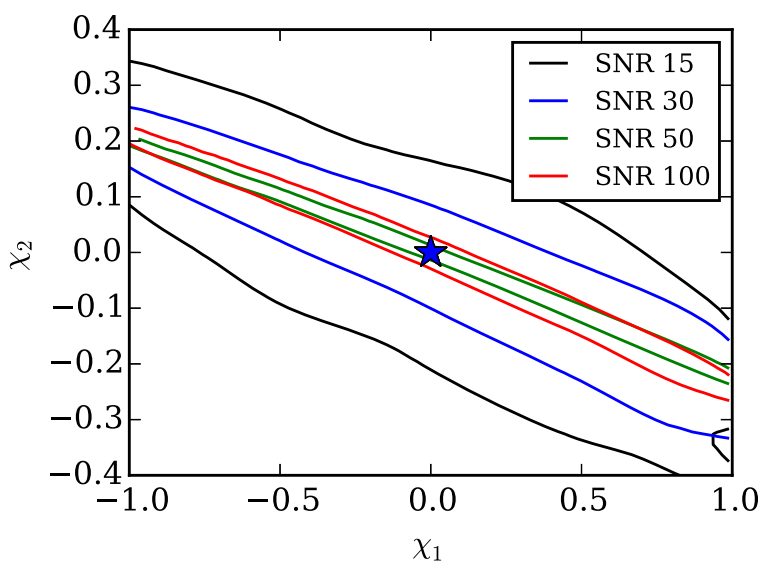

both contribute significantly. Figure 6 shows that this is indeed the case. At a total mass of $100 M_{\odot}$ the spin on the small black hole, $\chi_{1}$, can be somewhat constrained at SNR 100. Figure 7 illustrates how these results depend on the total mass. At SNR 30 (left) the spin on the small black hole, $\chi_{1}$, cannot be constrained at all, and at SNR 60 (middle) the lower bound starts to move away from -1 , while at SNR 100 (right) we see that the measurement accuracy for $\chi_{1}$ improves noticeably with the total mass until about $100 M_{\odot}$ and stays roughly the same until $200 M_{\odot}$

Systematic errors in the ROM and the underlying EOB model become important at high SNRs. In the region spanned by the posterior in Fig. 6 the ROM is only indistinguishable [39] from the original SEOBNRv2 waveform for SNRs of order $\sim 22$ for the worst mismatch against SEOBNRv2. With respect to the median ROM error this improves to a SNR of $\sim 55$. Parameter estimation at SNR 100 would require much more accurate models. However, even SEOBNRv2 is no longer indistinguishable against its NR calibration waveforms at such a high SNR. The indistinguishability criterion is sufficient, but not necessary, and it has been shown that it is in practice far too conservative where parameter estimation is concerned [40]. While these

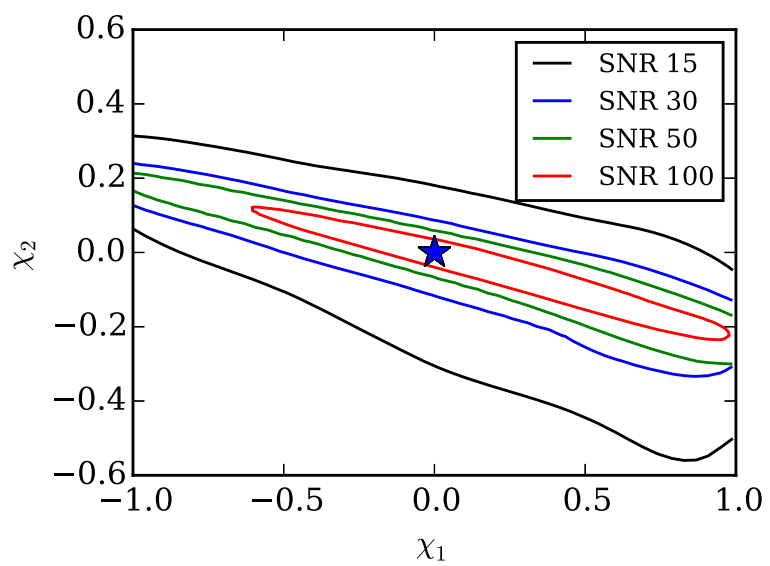

FIG. 6. The $95 \%$ joint credible regions of the component spins for a $q=4$ nonspinning system at a total mass of $12 M_{\odot}($ left) and $100 M_{\odot}$ (right) as a function of SNR.
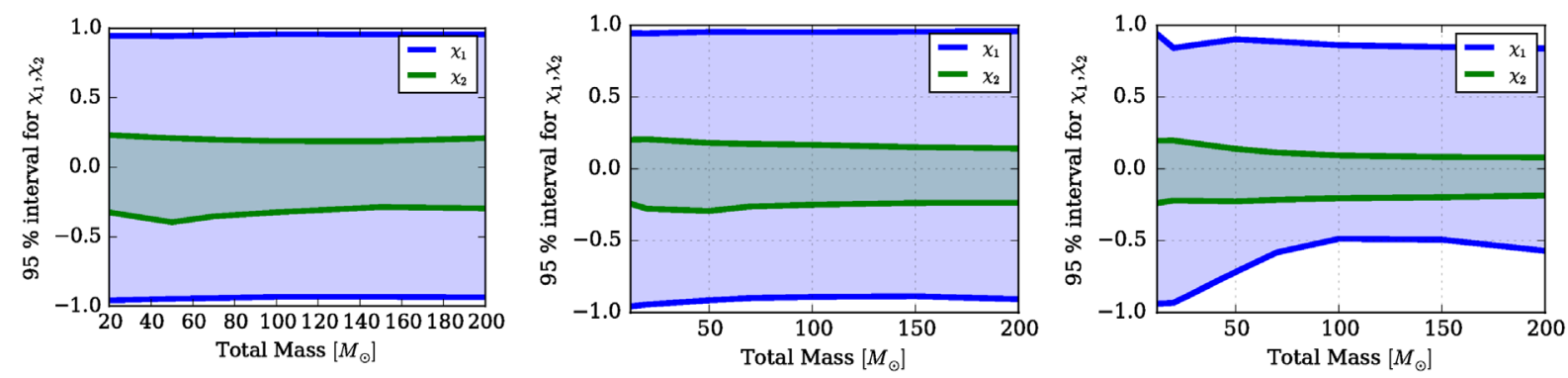

FIG. 7. The $95 \%$ credible intervals for the component spins for nonspinning binaries at mass ratio $q=4$ as a function of total mass. The panels show the spin on the small black hole (blue) and on the large black hole (green) for SNR 30 (left), SNR 60 (middle), and SNR 100 (right). 


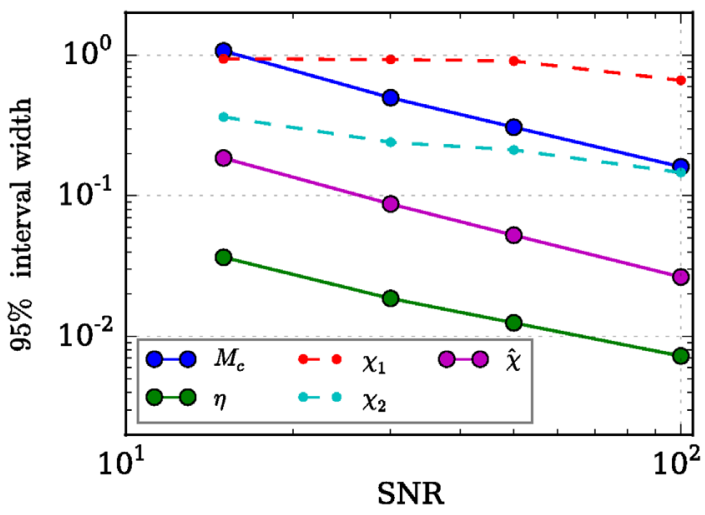

FIG. 8. The width of $95 \%$ credible intervals for chirp mass $M_{c}$, symmetric mass ratio $\eta$, rescaled reduced spin $\hat{\chi}$, and the alignedspin components $\chi_{1}, \chi_{2}$ against SNR. As in Fig. 6 results are shown for a nonspinning system at mass ratio $q=4$ at a total mass of $100 M_{\odot}$.

results should be taken with a grain of salt we want to make the point that if the ROM is a reasonably smooth model with respect to parameter variations, it can still be used even to give an estimate of the qualitative behavior of measurements at high SNR. We can sanity check the results in two ways: At low mass the credible regions obtained from the ROM agree with regions from IMRPhenomD, which fully incorporates two-spin effects during the early inspiral [30]. For sufficiently high SNR the posterior PDF is well approximated by a multivariate Gaussian and uncertainties on individual parameters scale inversely with SNR (see Fig. 8 and Fig. 7 of [34]). In Fig. 8 this scaling can already be seen at SNR 15.

These results suggest that the combined information from the inspiral and the merger-ringdown does indeed improve the measurement of the small black hole's spin, but this effect only becomes significant at SNRs of 100 or beyond. For such signals, a two-spin model can begin to place constraints on the spin of the smaller black hole, beyond what would already be known from a single-spin model and the consequences of the Kerr limit. For lower SNRs, however, we do not seem to recover significant extra information from the two-spin model. Note also that the improvement in the spin measurement is not strong-even for the strongest signals we expect to be able to detect with aLIGO at design sensitivity, we do not expect to be able to constrain the small black hole's spin to anything other than a statement that it is "small" or "large."

\section{DISCUSSION}

We have examined our ability to measure individual black-hole spins in aLIGO and AdV observations of aligned-spin black-hole-binary mergers. Our results are based on the SEOBNRv2 model, which aims to capture the effect of both black-hole spins on the GW signal. This model includes only the dominant $(\ell=2,|m|=2)$ harmonics and does not model precession effects. We will make some comments below on how we expect these approximations to affect our overall conclusions.

For low-mass binaries (less than $\sim 10 M_{\odot}$ ), we expect a degeneracy between the binary's mass ratio and a combination of the spins, and another degeneracy between the two spins themselves, to make it difficult to measure the individual spins (in particular the spin of the small black hole) below SNRs of $\mathcal{O}(1000)$. For higher masses, where the merger and ringdown contribute increasingly to the overall SNR, an alternative degeneracy (between systems with the same final mass and spin) dominates, and it is conceivable that observations that include both the inspiral and merger-ringdown will allow us to break these degeneracies and constrain measurements of both spins. That is what we have investigated here.

For the configurations that we have studied, this does not appear to happen until we reach SNRs of $\sim 100$. Below that the spins are constrained only by our knowledge of the extreme Kerr limit. This is illustrated in Fig. 1. For some configurations our ability to measure both spins is indeed optimal at masses where both the inspiral and mergerringdown contribute comparably to the total SNR (see Fig. 7), at masses of $\sim 100 M_{\odot}$, but this effect will only aid measurements of signals with very high SNR (see Fig. 6). We remind the reader that, assuming a uniform volume distribution of sources in the Universe, and a threshold detection SNR of $\sim 10$, SNRs above 100 will occur in roughly one in a thousand observations. The most optimistic current rate estimates [3,5] suggest that there might be several such observations per year when aLIGO and AdV reach their design sensitivity, but in these cases we would only be able to constrain the smallest black hole (or, in the case of equal-mass systems, both black holes) to possessing either "high" or "low" spin.

Our study is not exhaustive, but we have considered configurations over a wide sampling of the parameter space (up to mass ratios of $1: 10$ ). The results could change depending on the overall systematic accuracy of the SEOBNRv2 model. The model was found to be less accurate for mixed aligned and antialigned configurations [31] up to mass ratio $1: 3$. Beyond these mass ratios the model has not been systematically checked against unequal-spin waveforms. There are also known inaccuracies for high-aligned-spin systems near merger [30,31]. However, over much of the parameter space that we have considered, we expect the model to be robust, and we do not expect the qualitative picture to change.

The most important effect that we have not included in this study is precession. In configurations where the binary's total angular momentum is highly inclined with respect to the detector, strong modulations of the GW frequency and amplitude may be detectable. These add more structure to the waveforms. Some studies have shown how the inclusion of precession can improve measurements 

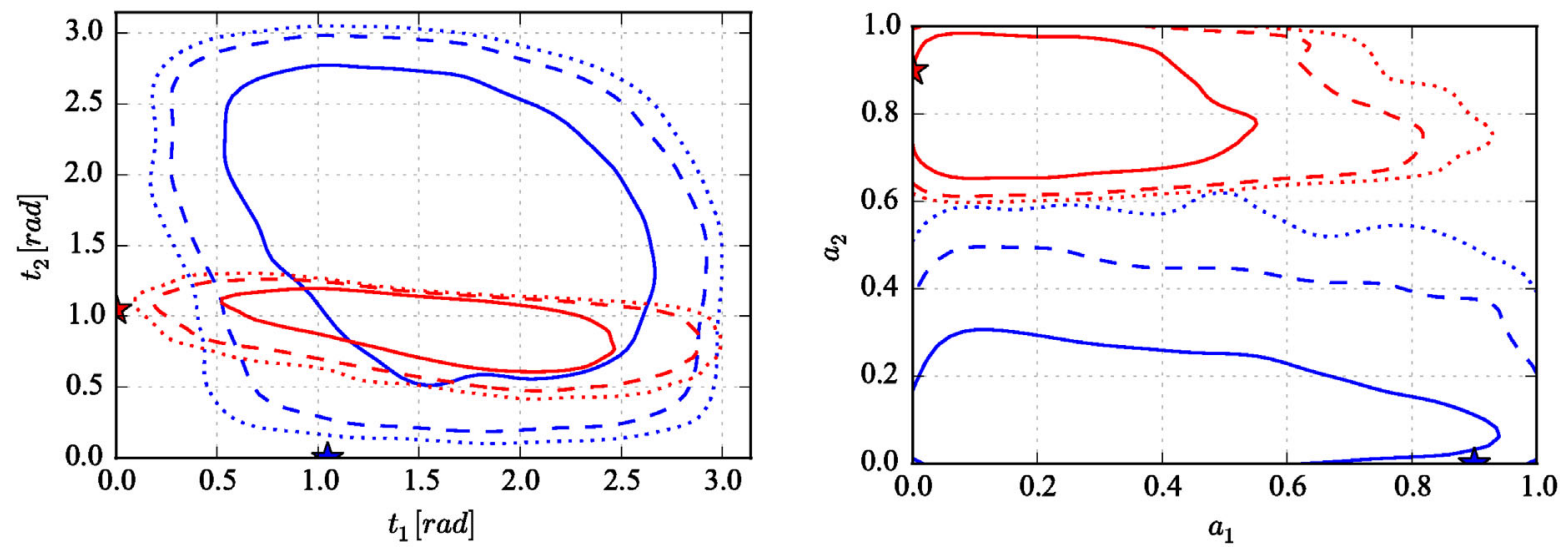

FIG. 9. The 68\%, 90\%, and 95\% credible regions (solid, dashed, and dotted lines, respectively) for the spin tilt angles (left) and spin magnitudes (right) for two configurations at $q=3$ and $M_{\text {tot }}=12 M_{\odot}$. We show credible regions for precessing spin on the small black hole (blue) or the large black hole (red). The true values of the spin magnitudes 0.9 and tilt angles $\pi / 3$ are indicated by stars.

of the mass ratio and the spin of the larger black hole [41-44], although there are still indications that it is only the spin of the larger black hole that can be measured with any reasonable accuracy $[13,45]$. To date no studies have explicitly considered our ability to measure individual spins or have considered the effects of merger and ringdown. For such systems, we note that we will observe only a small number of precession cycles, so the effect of precession on our measurements may be small. It should also be borne in mind that, if we measure the binary's parameters with a model with a larger number of physical parameters, we may well increase the size of the credible regions for each individual parameter; in this sense, the inclusion of precession may in some cases worsen our spin measurements.

As an illustration of the effects of precession on individual spin measurements, we consider an inspiralonly example, for which PN two-spin precessing models are available. We study two configurations with mass ratio
$1: 3$, a total mass of $12 M_{\odot}$, dimensionless spin magnitude 0.9 on either the small or the large black hole, with the other black hole nonspinning. The spin tilt angle is $t_{i}=\arccos \left(\hat{L} \cdot \hat{S}_{i}\right)=\pi / 3$, where $i$ refers to the spinning black hole. The binaries are viewed under an inclination of $3 \pi / 4$, at which we expect precession effects to be strong. We use SpinTaylorT4 [20] without amplitude corrections both for the signal and for recovery with a lower frequency cutoff of $f_{\text {low }}=40 \mathrm{~Hz}$ at SNR 30 and a sampling rate of $4096 \mathrm{~Hz}$. The simulations were performed with the nested sampling code from LALINFERENCE [23] for a three-detector aLIGO-AdV setup.

In Fig. 9 we compare the recovery of the spin magnitude and the tilt angles for the two configurations. If the spin is on the large black hole, its tilt angle can be recovered fairly accurately, while the tilt angle cannot be constrained if the spin is on the small black hole. The tilt angle of the nonspinning companion is not well defined for the signal and cannot be recovered. The spin magnitude can be
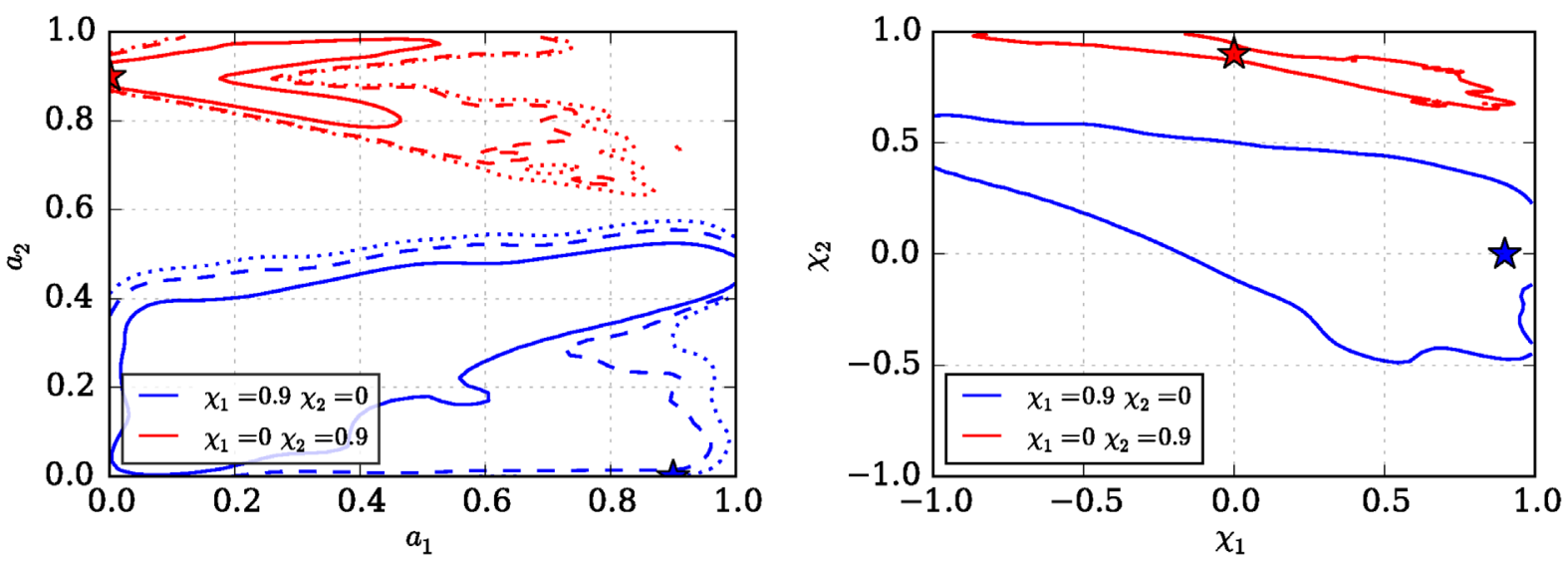

FIG. 10. The 68\%, 90\%, and 95\% credible regions (solid, dashed, and dotted lines, respectively) for aligned-spin SEOBNRv2-ROM configurations with $q=3$ and spin 0.9 on the small black hole (blue) or the large black hole (red). The left panel shows the spin magnitudes. The peculiar shape of the regions can be explained by the elongated regions in the component spins (right panel). 
recovered with some accuracy only if the larger black hole is spinning. Similar behavior has been seen for other configurations in $[45,46]$. Note that the prior on the spin tilt angles is uniform in the cosine of the tilt angles and, therefore, the true values do not appear inside the 95\% credible regions for the tilt angles.

Aligned spin comparison cases using SEOBNRv2_ ROM are shown in Fig. 10. These configurations use the same setup as the precessing simulations, in particular also $f_{\text {low }}=40 \mathrm{~Hz}$, except that the black holes carry aligned spin of +0.9 on the small or large black hole. The regions are elongated along the constant effective spin direction and by taking the modulus of the component spins the regions become V-shaped. We would expect to see a similar effect if we used a precessing-binary model in the parameter estimation, if we made the additional requirement that the tilt angles had to be close to either 0 or $\pi$. Apart from this notch the regions cover comparable area in the spin magnitudes as shown in Fig. 9 (right). The measurement of the individual spins has not improved at all in the precessing case.

Other effects we do not consider in this study are spherical harmonic modes beyond the dominant $(\ell=2,|m|=2)$ contributions. Higher harmonics become more important as the mass ratio is increased [35,40,47-49] and also add more structure to the waveforms. We would expect the inclusion of higher harmonics to improve the measurement of individual spins, and we once again have the possibility that there are ideal configurations (and binary orientations) that make it possible to accurately measure both spins. However, since in the present study we find that individual spins are very poorly constrained, and in most configurations the higher harmonics contribute less than a few percent of the total signal power, it seems unlikely that the situation will change dramatically. To address this question conclusively, of course, would require a two-spin higher-mode IMR model.

It is clear, then, that we require IMR models that include higher harmonics and precession in order to fully understand our potential to measure individual spins with advanced-detector observations. Even for models that include only the $(\ell=2,|m|=2)$ modes, we require far greater accuracy than currently available in order to fully quantify the accuracy of measurements from observations with SNRs greater than $\sim 30$. These qualifications aside, we expect that in general individual spins will be measurable only in cases where the spins are both near extremal, and (at least for aligned-spin binaries), both aligned or both antialigned with the orbital angular momentum.

\section{ACKNOWLEDGMENTS}

We thank John Veitch, Alex Nielsen, Vivien Raymond, Harald Pfeiffer, Prayush Kumar, Alessandra Buonanno, Guillaume Faye, Salvatore Vitale, Eric Poisson and Aaron Zimmerman for useful discussions and comments. We thank Edward Fauchon-Jones for contributions at the early stages of the project. M. H. was supported by Science and Technology Facilities Council Grant No. ST/H008438/1, M. H. and F. O. were supported by European Research Council Consolidator Grant No. 647839 and M. P., M. H. and F. O. by ST/I001085/1. M. P. also thanks the Max Planck Gesellschaft for support. LALINFERENCE simulations were carried out at Advanced Research Computing (ARCCA) at Cardiff.

Note added.-Recently, the LIGO and Virgo Collaborations reported a gravitational-wave observation of a black-hole binary [50]. The spin measurements reported for that observation are consistent with the expectations presented here [51].
[1] J. Aasi et al. (LIGO Scientific Collaboration), Classical Quantum Gravity 32, 115012 (2015).

[2] F. Acernese et al. (VIRGO Collaboration), Classical Quantum Gravity 32, 024001 (2015).

[3] J. Abadie et al. (VIRGO, LIGO Scientific Collaboration), Classical Quantum Gravity 27, 173001 (2010).

[4] J. Aasi et al. (LIGO Scientific, VIRGO Collaborations), Living Rev. Relativity 19, 1 (2016).

[5] M. Dominik, E. Berti, R. O'Shaughnessy, I. Mandel, K. Belczynski, C. Fryer, D. Holz, T. Bulik, and F. Pannarale, Astrophys. J. 806, 263 (2015).

[6] C. Cutler and E. E. Flanagan, Phys. Rev. D 49, 2658 (1994).

[7] E. Poisson and C. M. Will, Phys. Rev. D 52, 848 (1995).

[8] E. Baird, S. Fairhurst, M. Hannam, and P. Murphy, Phys. Rev. D 87, 024035 (2013).
[9] P. Ajith, Phys. Rev. D 84, 084037 (2011).

[10] F. Ohme, A. B. Nielsen, D. Keppel, and A. Lundgren, Phys. Rev. D 88, 042002 (2013).

[11] T. A. Apostolatos, C. Cutler, G. J. Sussman, and K. S. Thorne, Phys. Rev. D 49, 6274 (1994).

[12] L. E. Kidder, Phys. Rev. D 52, 821 (1995).

[13] P. Schmidt, F. Ohme, and M. Hannam, Phys. Rev. D 91, 024043 (2015).

[14] M. Pürrer, M. Hannam, P. Ajith, and S. Husa, Phys. Rev. D 88, 064007 (2013).

[15] A. Taracchini et al., Phys. Rev. D 89, 061502 (2014).

[16] LIGO Scientific Collaboration, Technical Report No. LIGO-T0900288-v3, LIGO Project, 2009, https://dcc .ligo.org/DocDB/0002/T0900288/003/AdvLIGO\%20noise $\% 20$ curves.pdf. 
[17] L. S. Finn and D. F. Chernoff, Phys. Rev. D 47, 2198 (1993).

[18] W. Farr, LIGO Technical Report No. LIGO-T1400460, 2014.

[19] D. Foreman-Mackey, D. W. Hogg, D. Lang, and J. Goodman, Publ. Astron. Soc. Pac. 125, 306 (2013).

[20] LSC Algorithm Library (LAL), LIGO Scientific Collaboration, https://wiki.ligo.org/DASWG/LALSuite.

[21] C. Capano, I. Harry, S. Privitera, and A. Buonanno, arXiv:1602.03509.

[22] A. Gelman and D. B. Rubin, Stat. Sci. 7, 457 (1992).

[23] J. Veitch et al., Phys. Rev. D 91, 042003 (2015).

[24] A. Manzotti and A. Dietz, arXiv:1202.4031.

[25] M. Pürrer, Phys. Rev. D 93, 064041 (2016).

[26] M. Pürrer, Classical Quantum Gravity 31, 195010 (2014).

[27] http://www.black-holes.org/waveforms.

[28] S. Husa, S. Khan, M. Hannam, M. Pürrer, F. Ohme, X. J. Forteza, and A. Bohé, Phys. Rev. D 93, 044006 (2016).

[29] T. Chu, H. Fong, P. Kumar, H. P. Pfeiffer, M. Boyle, C. Hadmer, D. Hemberger, L. E. Kidder, M. A. Scheel, and B. Szilagyi, Report No. LIGO-P1500250.

[30] S. Khan, S. Husa, M. Hannam, F. Ohme, M. Pürrer, X. J. Forteza, and A. Bohé, Phys. Rev. D 93, 044007 (2016).

[31] P. Kumar, T. Chu, H. P. Pfeiffer, M. Boyle, C. Hadmer, D. Hemberger, L. Kidder, M. A. Scheel, and B. Szilagyi, Report No. LIGO-P1500251.

[32] M. Hannam, P. Schmidt, A. Bohé, L. Haegel, S. Husa, F. Ohme, G. Pratten, and M. Pürrer, Phys. Rev. Lett. 113, 151101 (2014).

[33] M. Hannam, D. A. Brown, S. Fairhurst, C. L. Fryer, and I. W. Harry, Astrophys. J. 766, L14 (2013).

[34] J. Veitch, M. Pürrer, and I. Mandel, Phys. Rev. Lett. 115, 141101 (2015).
[35] P. B. Graff, A. Buonanno, and B. Sathyaprakash, Phys. Rev. D 92, 022002 (2015).

[36] C.-J. Haster, Z. Wang, C. P. L. Berry, S. Stevenson, J. Veitch, and I. Mandel, Mon. Not. R. Astron. Soc. 457, 4499 (2016).

[37] M. Vallisneri, Phys. Rev. D 77, 042001 (2008).

[38] A. B. Nielsen, Classical Quantum Gravity 30, 075023 (2013).

[39] L. Lindblom, B. J. Owen, and D. A. Brown, Phys. Rev. D 78, 124020 (2008).

[40] T. B. Littenberg, J. G. Baker, A. Buonanno, and B. J. Kelly, Phys. Rev. D 87, 104003 (2013).

[41] K. Chatziioannou, N. Cornish, A. Klein, and N. Yunes, Astrophys. J. 798, L17 (2015).

[42] R. O'Shaughnessy, B. Farr, E. Ochsner, H.-S. Cho, V. Raymond, C. Kim, and C.-H. Lee, Phys. Rev. D 89, 102005 (2014).

[43] T. B. Littenberg, B. Farr, S. Coughlin, V. Kalogera, and D. E. Holz, Astrophys. J. 807, L24 (2015).

[44] B. Farr et al., arXiv:1508.05336.

[45] S. Vitale, R. Lynch, J. Veitch, V. Raymond, and R. Sturani, Phys. Rev. Lett. 112, 251101 (2014).

[46] S. Vitale (private communication).

[47] V. Varma, P. Ajith, S. Husa, J. C. Bustillo, M. Hannam, and M. Pürrer, Phys. Rev. D 90, 124004 (2014).

[48] M. Wade, J. D. E. Creighton, E. Ochsner, and A. B. Nielsen, Phys. Rev. D 88, 083002 (2013).

[49] C. Capano, Y. Pan, and A. Buonanno, Phys. Rev. D 89, 102003 (2014).

[50] B. P. Abbott et al. (Virgo, LIGO Scientific Collaboration), Phys. Rev. Lett. 116, 061102 (2016).

[51] B. P. Abbott et al. (Virgo, LIGO Scientific Collaboration), arXiv:1602.03840. 